\title{
New Tools in Experimental Cellular Therapy for the Treatment of Liver Diseases
}

\author{
Jennifer R. Ferrer ${ }^{1,2,3,4,5}$ • Attasit Chokechanachaisakul ${ }^{1,6}$ • Jason A. Wertheim ${ }^{1,3,4,5,6,7,8}$
}

Published online: 15 April 2015

(C) Springer International Publishing AG 2015

\begin{abstract}
The current standard of care for end stage liver disease is orthotopic liver transplantation (OLT). Through improvement in surgical techniques, immunosuppression, and general medical care, liver transplantation has become an effective treatment over the course of the last half-century. Unfortunately, due to the limited availability of donor organs, there is a finite limit to the number of patients who will benefit from this therapy. This review will discuss current research in experimental cellular therapies for acute, chronic, and metabolic liver failure that may be appropriate when liver transplantation is not an immediate option.
\end{abstract}

This article is part of the Topical Collection on Cellular Transplants

Jason A. Wertheim

jason.wertheim@northwestern.edu

1 Department of Surgery, Northwestern University Feinberg School of Medicine, Chicago, IL 60611, USA

2 Department of Chemistry, Northwestern University, Evanston, IL 60201, USA

3 Chemistry of Life Processes Institute, Northwestern University, Evanston, IL 60201, USA

4 International Institute for Nanotechnology, Northwestern University, Evanston, IL 60201, USA

5 Simpson Querrey Institute for BioNanotechnology in Medicine, Northwestern University, Chicago, IL 60611, USA

6 Comprehensive Transplant Center, Feinberg School of Medicine, Northwestern University, 676 St. Clair St. Suite 1900, Chicago, IL 60611, USA

7 Department of Surgery, Jesse Brown VA Medical Center, Chicago, IL 60612, USA

8 Department of Biomedical Engineering, Northwestern University, Evanston, IL 60201, USA
Keywords Hepatocyte transplantation · Cellular therapy · Alternative cell sources $\cdot$ Stem cells

\section{Introduction}

A healthy liver is remarkable in its intrinsic ability to regenerate and heal itself in response to a wide range of injurious stimuli. However, when liver health is compromised by viral infection, metabolic deficiency, genetic abnormality, or alcohol abuse, the regenerative capacity of the liver is diminished. For many people worldwide, this leads to development of acute liver disease (ALD), metabolic liver disease or chronic liver disease leading to end-stage liver disease (ESLD). For a patient with ESLD of any etiology, the standard of care for over 30 years has been orthotopic liver transplantation (OLT), either deceased donor liver transplant (DDLT) or living donor liver transplant (LDLT). OLT is the only curative means of treating ALD, chronic liver disease, and inborn metabolic disease by effectively replacing the failing liver with a healthy liver graft. However, the number of available liver donors limits this life-saving surgical procedure. Despite improvements in surgical technique, such as split-liver and livingrelated liver donor grafts, and efforts to increase the donor pool by broadening donor criteria, the number of organ transplants performed annually has remained stagnant over the last 10 years while the number of patients in need of a liver transplant continues to grow. Therefore, alternatives to liver transplantation must be considered and further developed.

Hepatocyte transplantation (HT) is one such alternative that focuses on reconstituting the liver parenchyma as opposed to outright replacing it. HT requires the isolation of human hepatocytes from livers unsuitable for OLT and subsequent transplantation of cells into patients with liver disease via portal vein injection or injection into the spleen [1-3]. Due to the 
high number of cells needed to achieve a therapeutic effect and high risk of portal vein thrombosis, HT generally requires multiple infusions of donor cells $[4,5]$. Nonetheless, this approach is far less invasive than OLT and bypasses many of the complications associated with such an invasive surgery, such as risk of infection, biliary stricture/leak, excessive bleeding, and cardiac injury. This makes hepatocyte transplantation a potentially safer alternative, especially for high-risk patients. To date, at least 100 patients have successfully received a hepatocyte transplant worldwide [6-8]. HT is mainly used to correct enzyme deficiencies in children with inborn metabolic disease, which is a small component of liver diseases that necessitate OLT (Table 1). HT can reverse hepatic failure in acute liver disease; however, only partial correction of metabolic dysfunction is often achieved, and treating chronic liver disease with HT has not demonstrated significant success due to the more complicated nature of ESLD [10]. Numerous clinical trials validated the long-term safety of HT [11-14]. However, there remains several limitations that prevent HT from becoming a widely used therapy, despite its advantage as a less invasive and potentially safer alternative to OLT. The major limitations associated with HT are the short lifespan of isolated hepatocytes, low engraftment efficiency, variable hepatocyte quality, and an inability to expand primary hepatocytes ex vivo.

\section{Barriers to Successful Primary Hepatocyte Transplantation}

HT generally requires multiple infusions to achieve therapeutic efficacy. However, cold storage of hepatocytes requires optimal conditions to maintain high numbers of viable, minimally damaged cells diminishing the ability to bank cells for transplantation. The standard storage condition for freshly isolated hepatocytes is below $4{ }^{\circ} \mathrm{C}$. At this temperature, metabolism slows, but cellular damage remains inevitable. Gramignoli et al. showed that immediately following isolation, cell viability is $\sim 80 \%$. After $24 \mathrm{~h}$ in cold storage, viability drops to $60 \%$ and after $72 \mathrm{~h}$, less than $50 \%$ of cells remain viable [15]. Although cell viability decreased over time,

Table 1 Summary of primary cause of liver disease treated by liver transplantation

\begin{tabular}{ll}
\hline Primary cause of disease & Percentage \\
\hline Hepatitis C virus (HCV) & 24.5 \\
Malignancy & 22.4 \\
Alcoholic liver disease & 17.2 \\
Cholestatic disease & 8 \\
Acute hepatic necrosis & 3.7 \\
Metabolic liver disease & 2.4 \\
Other & 22 \\
\hline
\end{tabular}

Table adapted from [9] functionality appeared normal, as measured by ability to attach to an extracellular matrix cell culture substrate, metabolize ammonia, and express the CYP 1A, 3A, and 2C family of P450 enzymes. Freshly isolated hepatocytes can be cryopreserved for later use; however, cryopreservation leads to lower engraftment efficiency compared to fresh hepatocytes. Therefore, development of methods using new storage solutions and conditions to improve cold storage of hepatocytes need to be further explored and validated [16].

Engraftment efficiency is a difficult marker to assess and can only be gauged in retrospect. Roll et al. demonstrated that injection of nonviable human hepatocytes into mice led to detection of human albumin in mouse serum that remained detectable for over a week [17]. These results confound standard analysis of engraftment efficiency, as albumin production is often used as a marker of hepatocyte graft function. Other factors that may influence engraftment include duration after cell isolation, number of transplanted donor cells, health and viability of donor cells, and donor-recipient matching and compatibility. Although animal models used to assess human hepatocyte transplantation strategies are useful, many may not truly represent the engraftment process in humans [18]. Methods to improve cell engraftment, such as liver preconditioning, hepatic irradiation, and portal embolization, have been successful in animal models and are being validated for human application [19-21]. Extra-hepatic implantation sites, such as the spleen, have also been investigated as engraftment sites and many studies demonstrated that injected hepatocytes home to and colonize the spleen [22, 23•]. Often times, the ectopic transplantation site is capable of compensating for primary liver failure, but the frequency depends on the number of transplanted cells that eventually engraft into the ectopic site.

The major hurdle to successful HT is isolation of sufficient quantity of cells with high potency. For some cases of metabolic liver disease, replacing less than $10 \%$ of the liver volume with transplanted hepatocytes may lead to a degree of clinical benefit $[4,6]$. For most cases, however, at least 20 $30 \%$ of the liver volume will need to be augmented by transplanted hepatocytes [24]. Nonetheless, primary adult hepatocyte transplantation has a known benefit [8]. When compared with fetal hepatocytes and embryonic stem cell-derived endodermal cells in an ALD mouse model, adult hepatocytes had the highest survival rate and proliferative capacity at 35 days post transplantation [25]. It is possible to increase donor cell number by mixing hepatocytes isolated from livers removed during OLT for metabolic disease. Hepatocytes from these livers, aside from a deficiency in a single enzyme, are functional hepatocytes. In comparison to hepatocytes isolated from a healthy donor liver, hepatocytes from metabolic diseased livers demonstrate similar cell viability, plating efficiency, morphology, and performance in assays of liver function, such as ammonia metabolism, drug metabolism, and 
conjugation reactions [26]. Additionally, hepatocytes with a metabolic deficiency were able to repopulate an FRG mouse liver with 50-99 \% efficiency and produced human albumin. Primary hepatocytes can also be modified to induce immortality thus allowing ex vivo expansion. Meng et al. isolated primary human hepatocytes and transduced them with a retroviral vector containing SV40T and a hygromycin resistant gene. Though SV40T induced immortality, there remain concerns over the use of viral vectors in the clinical setting due to possible expression of viral genes in transfected cells. To address this concern, the SV40T gene cDNA was excised using Cre/loxP site-specific recombination in isolated, expanded clones [27]. This method makes it possible to expand human hepatocytes ex vivo and remove their proliferative capacity before transplantation.

Although primary human hepatocytes have demonstrated clinical safety and success in a limited number of cases, the aforementioned limitations prevent their widespread use as a therapy. Hepatocyte transplantation requires large numbers of donor cells in order to be effective. Alternative cell sources, namely stem and progenitor cells, are now being investigated and address issues of expansion, variable donor quality, the requirement of immunosuppression, and longevity.

\section{Alternative Cell Sources for Hepatocyte Transplantation}

\section{Liver Stem/Progenitor Cells}

Liver progenitor cells, also called hepatic oval cells, are bipotential cells that are activated and expand in the liver parenchyma when mature hepatocytes are exposed to liver injury, thus playing a major role in liver repair and regeneration (Fig. 1). These cells are present in low numbers in a healthy liver, but are capable of differentiating into either hepatocytes or biliary cells and compensate for liver function when hepatocytes and biliary cells are impaired [28, 29]. These cells are a potential experimental cell source for HT because liver progenitor cells can be isolated and stimulated ex vivo to differentiate into mature hepatocytes [30, 31]. Alternatively, progenitor cells can be recovered from livers with chronic liver injury and potentially expanded ex vivo for cell therapy applications. Using a mouse model of oval cell activation, Tarlow et al. discovered that both human and mouse hepatocytes undergo a sort of dedifferentiation to a progenitor state that can then be reversed [32•]. However, alternative models of hepatocyte proliferation in response to injury leading to liver regeneration have been recently proposed and suggest that mature hepatocytes participate in the repair process $[33,34]$.

\section{Mesenchymal Stem Cells}

Mesenchymal stem cells (MSCs) are of extra-hepatic origin and are adherent, fibroblast-like non-hematopoietic progenitor cells. MSCs are a population of stem cells that are being explored experimentally for regenerative purposes due to their multipotency. Most research involves the use of bone marrowderived MSCs (BM-MSCs), but MSCs can be retrieved from other tissues and organs, including the umbilical cord and subcutaneous adipose tissue [35-38]. Recent evidence suggests that MSCs are capable of sensing injury and such signals promote MSC migration to injury sites and facilitate repair or regeneration. There is overlap between the ability of liver stem cells and BM-MSCs to induce liver regeneration [39, 40]. The use of MSCs as a cellular therapy for liver disease has been demonstrated in both animal models and in limited clinical studies. In one study, human umbilical cord blood-derived MSCs (hUCBMSs) were transplanted via tail vein injection into SCID mice with D-galactoasmine/LPS induced liver injury. hUCBMSCs differentiated into hepatocyte-like cells that homed to the liver and attenuated liver damage, as assessed by
Fig. 1 Description of cell sources that have been investigated for use in hepatocyte transplantation

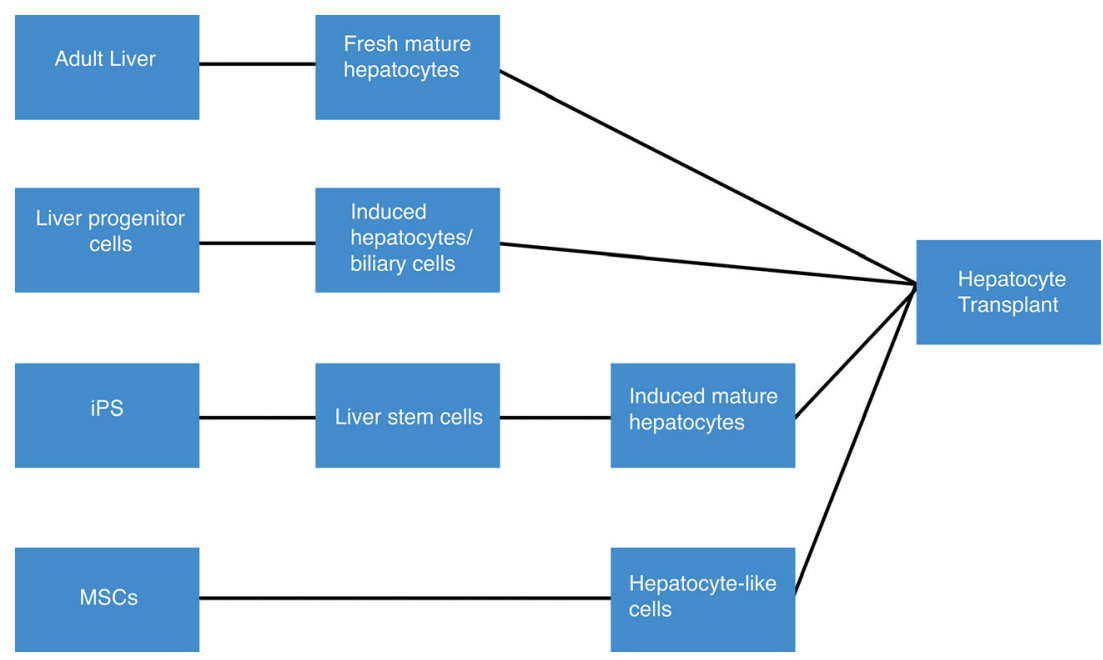


H\&E, which demonstrated resolution of liver damage, disappearance of inflammatory cell infiltration, and decreased necrosis [41]. In a clinical setting, 40 patients with hepatitis $\mathrm{C}$ were divided into two groups, with one group receiving BMMSCs either by intrasplenic or intrahepatic transplantation, while the other group received traditional supportive treatment for Hepatitis $\mathrm{C}$ virus. The results of this study, which followed patients for 6 months, showed improvement in MELD scores, Child-Pugh scores, and serum albumin levels in patients that received the MSC transplant, suggesting that BM-MSCs may be safe and effective for the treatment of liver failure [42].

\section{Induced Pluripotent Stem Cells}

Induced pluripotent stem (iPS) cells are derived from terminally differentiated cells, such as skin fibroblasts, and are made pluripotent by expression of specific transcription factors [43]. These cells represent a potentially ideal source of hepatocytes for transplantation in that these cells can be generated in a patient-specific manner, potentially negating the need for immunosuppression. iPS-derived hepatocytes (iPSheps) have been generated from a range of somatic cells from various donors, and any variation in differentiated iPS-heps, interestingly, can be attributed to donor differences rather than the initial cell type used, suggesting that iPS-heps may be a more homogenous population of cells than freshly isolated hepatocytes [44, 45]. A number of studies have tested the efficacy of liver repopulation using human iPS-derived hepatocytes in mouse models. In one study, wild-type iPS cells were injected into fumarylacetoacetate hydrolase (FAH) knockout mouse blastocysts. FAH $-/-$ mice lack the fumaryl-acetoacetate hydrolase enzyme, and this deficiency is a model for tyrosinemia type I [46]. These mice consistently develop liver disease when a protective drug that prevents tyrosine degradation, 2-(2-nitro-4-trifluoromethylbenzoyl)-1, 3 -cyclohexanedione (NTBC), is withdrawn. The FAH -/blastocysts complemented with wild-type iPS cells were then transferred into foster mothers, and chimeric neonates showed growth and survival even when NTBC was withdrawn, suggesting hepatocytes derived from FAH +/+ iPS cells were functional and could compensate for the liver disease that is lethal to neonates [47]. In another study, rather than generate true iPS cells, the authors transformed human fibroblasts into multipotent progenitor cells from which hepatocyte-like cells could be generated. These induced multipotent progenitorheps, unlike iPS-heps, proliferated extensively and demonstrated hepatocyte function similar to human adult hepatocytes when transplanted into an immune-deficient mouse model of human liver failure [48•]. These results are particularly impressive, and suggest that a large number of patientspecific hepatocyte-like cells can be generated in vitro. One limitation to this strategy, and other protocols developing iPS cells and iPS-heps, is the time scale required to generate mature hepatocytes and their limited functionality compared to primary human hepatocytes. In general, it takes at least 3 weeks to generate iPS cells and differentiate them toward a hepatocyte-like lineage $[45,49,50]$. However, Tomizawa et al. developed a protocol to differentiate iPS cells into iPSheps in eight days. iPS cells were exposed to a combination of oncostatin $\mathrm{M}$, epidermal growth factor, retinoic acid, dexamethasone, and ITS, supplemented with various growth factors. iPS cells were hepatocyte-like in their expression of GATA4, GATA6, Sox17, FoxA2, HNF4a, but did not produce albumin [51]. As albumin production is a hallmark of hepatocyte function, this strategy, though more expedient than other protocols, requires additional investigation.

\section{Other Cell Sources}

Other cell sources that have been explored as potential cellular therapies for liver disease include embryonic stem cells, a human hepatoma cell line, xenogeneic hepatocytes, and humanized mouse hepatocytes [52-58]. These cell types will not be described in detail in this review, however, it should be noted that xenogeneic tissue and humanized animal hepatocytes have the greatest potential to address the donor limitation of primary human hepatocytes, but issues regarding tissue matching and zoonosis remain.

\section{Hepatocyte Transplantation into Animal Models of Liver Disease}

In order to gauge the efficacy of hepatocytes and other cell sources for transplantation, animal models that accurately recapitulate human liver diseases are desperately needed. Small rodents, especially mice, are an attractive animal model because of their ease of handling, short life span, and broad genetic similarity. The development of numerous animal models of liver disease has led to a new understanding of the mechanism of liver repair and regeneration in recent years.

\section{Metabolic Liver Disease}

Although HT is not yet a widely used therapy, it has been most successful in treatment of inborn metabolic disorders. There are several animal models of metabolic liver disease, including the Gunn rat model of Crigler-Najjar syndrome, the Watanabe rabbit for familial hypercholesterolemia, and the FAH models of hereditary tyrosinemia $[59-62,63 \bullet \bullet]$. Crigler-Najjar syndrome is an autosomal recessive disorder that is characterized by low expression or absence of the enzyme uridine diphosphate glucuronosyltransferase. This mutation prevents patients with Crigler-Najjar syndrome from completely metabolizing bilirubin, resulting in jaundice and potential brain damage. The Gunn rat lacks the uridine 
diphosphate glucuronosyltransferase enzyme, is also jaundiced, and has been used for decades to study Crigler-Najjar syndrome. Recently, Maerckx et al. showed that transplantation of differentiated human liver progenitor cells into the Gunn rat corrects for the enzyme deficiency and lowers serum bilirubin levels [64]. In their study, hepatocytes isolated from a healthy male donor post-mortem expanded and differentiated ex vivo into mature hepatocyte-like cells with CYP3A4 activity. These cells were injected into the portal vein of Gunn rats, and serum bilirubin levels were assessed at multiple time points. Compared with rats that received a transplantation of cryopreserved hepatocytes, rats that received the differentiated progenitor cells had a similar reduction in serum bilirubin over a period of 27 weeks. Although HT has already been used to successfully treat patients with Crigler-Najjar syndrome, the Gunn rat remains a valuable tool for developing and optimizing strategies for HT as well as for testing the performance of alternative cell sources.

Hereditary tyrosinemia is an autosomal recessive disease that is caused by a deficiency in the FAH enzyme, which catalyzes the last step of tyrosine metabolism. This deficiency results in buildup of toxic metabolic byproducts that may lead to death if left untreated. The FAH knockout mouse is an excellent tool for studying hereditary tyrosinemia and for developing cellular therapies for this disease. In this model, both allogeneic BM-MSC and xenogeneic hepatocyte transplantation can successfully repopulate the mouse liver and rescue the enzyme deficiency (Fig. 2) [66, 67]. More recently, hepatic progenitor-like cells have been used as a cell source for HT into this animal model. In one study, mouse embryonic fibroblasts were reverted to a hepatic progenitor state by the transduction of two liver liver-specific transcription factors, HNF1 $\beta$ and Foxa3, important for liver organogenesis. When these cells were cultured in vitro on different substrates, they differentiated into both hepatocytes and cholangiocytes. When the stem cells were transplanted into the spleen of FAH knockout mice, they populated the liver and differentiated into hepatocytes and restored liver function [68]. In another study, fetal fibroblasts were isolated from pregnant FAH knockout mice and reprogrammed into iPS cells by transducing Oct4, Sox2, Klf4, and c-Myc transcription factors. The FAH mutation was corrected in these iPS cells by transfecting the cells with a viral vector expressing wild-type FAH cDNA.
The genetically corrected cells were complemented with tetraploid embryos and transferred into pseudopregnant FAH knockout mothers. Almost $25 \%$ of complemented embryos developed into viable pups [69]. The results from both of these studies are promising and exciting because iPS cells can be derived in a patient-specific manner, possibly bypassing the need for immunosuppressants. Even patients with metabolic disease can potentially benefit from self-derived iPS cells if gene correction can be done efficiently. However, testing the efficacy of gene correction of human iPS cells poses a limitation, as tetraploid embryo complementation cannot be tested in humans.

There are additional animal models of inborn metabolic liver diseases that have been successfully treated by HT [70-74]. Unlike models of acute and chronic liver disease, it is easier to recapitulate the etiology and presentation of metabolic liver disease in animals because the disease is caused by a gene mutation resulting in deficiency of a single enzyme. The FAH knockout pig by Hickey et al. is an especially important development because there are few large animal models of liver disease, and large animal models provide another layer of therapy validation in advance of human studies [63••]. The development and use of these models to validate HT has and will continue to be critical to its translation into the clinic.

\section{Acute Liver Disease}

Acute liver failure (ALF) is a severe liver disease with rapid onset and very high mortality. Only about $40 \%$ of patients with fulminant liver failure can be treated with medical management, and in those cases, it is the regenerative ability of the liver that allows the organ to recover, avoiding the need for OLT. A barrier to treating ALF with HT is that patients require transplantation of healthy hepatocytes that function immediately to correct liver failure [75]. For this reason, Sun et al. asserts that BM-MSCs are an appropriate choice for HT [76]. In a Concanavalin A-induced mouse model of ALF, Sun et al. found that only BM-MSC injection into the spleen (and not adult hepatocytes, induced hepatocytes or fetal liver cells) were capable of partially rescuing animals with liver failure, as observed by a reduction in the inflammatory response and overall prolonged survival. However, the efficacy of a cell-

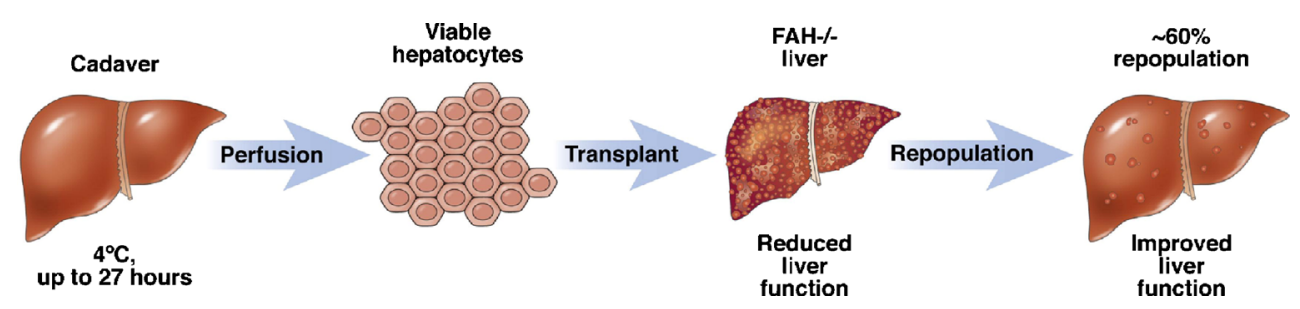

Fig. 2 Isolation of primary human hepatocytes and implantation into a FAH -/- recipient allows for repopulation and restoration of liver function. (Reproduced with permission from [65] 
based therapy seems to vary with the choice of animal model. In one study that used diphtheria toxin to induce ALF in mice, transplanted adult hepatocytes showed higher engraftment and survival rates as well as proliferation when compared to fetal liver cells and ESC-derived endodermal cells while yet another model of ALF (using LPS and D-galactosamine in rats) found that BM-MSCs homed to the liver and improved liver function $[25,77]$.

Another animal model, the Long-Evans Cinnamon (LEC) rat, spontaneously develops fulminant hepatitis during old age (80-120 days), and acute hepatic failure occurs due to copper accumulation from incomplete deletion of the copper transporter gene (Atp7b). This model more closely relates to Wilson's Disease, an autosomal recessive genetic disorder characterized by a disruption of copper metabolism leading to copper accumulation in the liver. But in the context of ALF, one study used LEC rats fed with a high-copper diet to rapidly induce liver failure. These animals were transplanted with Long-Evans agouti rat wild-type hepatocytes. Even in the presence of continued oral intake of copper in the diet, multiple rounds of infusions of adult hepatocytes into the spleen were required to delay onset of hepatitis ( $40 \%$ rats), normalize hepatitis-associated serum markers and prolong survival until the end of the study, while rats with one or no infusion of hepatocytes failed to survive past 22 days [78].

There are a variety of animal models that mimic the onset and symptoms of ALF that are induced surgically, pharmacologically, or through a combination of each [79, 80]. Perhaps the most promising application of BM-MSCs has been the work of $\mathrm{Li}$ et al. in which a porcine model of ALF was used [81•]. While small animal models are useful and can be analyzed quickly with high-throughput, large animal models hold more value in translating potential therapies. In the study by $\mathrm{Li}$ et al., ALF was induced by D-galactosamine, and human BMMSCs were transplanted into pigs via the intra-portal route or peripheral vein injection immediately followed by D-galactosamine administration. Animals that did not receive HT and animals given HT via a peripheral vein died within $96 \mathrm{~h}$. Animals that received intra-portal injection of human MSCs survived longer than 6 months. These results suggest that MSCs immediately begin participating in the regenerative and restorative process to overcome drug-induced ALF. Additionally, treated animals were healthy, suggesting that human BMMSC transplantation may be safe and effective in a large animal model of ALF and may be promising as a future clinical therapy.

\section{Chronic Liver Disease}

Chronic liver disease (CLD) is generally characterized by the presence of inflammation, liver fibrosis, and cirrhosis. Treating chronic liver disease with HT is even more difficult to treat than ALD or inborn metabolic diseases due to the dysregulated architecture of the liver. Excessive extracellular matrix protein accumulation makes HT difficult because blood vessels, like the portal vein, may experience severe hypertension when a bolus of hepatocytes is infused [75]. However, extrahepatic sites, like the spleen and lymph nodes, have the potential to compensate for liver failure if the ectopic sites are colonized or engrafted with sufficient number of cells. Using this strategy, HT in animal models of cirrhosis has shown improvement in liver function, but success in humans has been suboptimal, perhaps due to an insufficient cell load needed to compensate for severely deficient liver function [82-84]. Like ALF, there are a number of surgical and pharmacological models for CLD, mostly using mice and rats $[85,86]$. The most common model uses carbon tetrachloride $(\mathrm{CCl} 4)$, though thioacetamide and dimethylnitrosamine are often used as well. Though these toxin-induced models do not necessarily replicate the etiology of CLD, they do provide useful tools for assessing the effect of HT on modulating inflammation and fibrosis.

BM-MSCs are an attractive cell source for treating CLD because of their ability to differentiate into hepatocyte-like cells, their regenerative capabilities, and their antiinflammatory properties. In CCl4-induced mouse models of fibrosis and cirrhosis, undifferentiated and differentiated BMMSCs engraft in the liver and effectively increase liver function and health, as measured by expression of hepatocytespecific markers, albumin production, decreased fibrosis and total bilirubin levels. Additionally, BM-MSCs increase the survival rates of CCl4-induced mouse models of liver fibrosis and cirrhosis $[87,88]$. Piryaei et al. asserts that in their work, BM-MSCs differentiated into hepatocyte-like cells and functioned better than undifferentiated cells. In an immunodeficient mouse model of non-alcoholic steatohepatitis (NASH), Winkler et al. demonstrated both the regenerative potential of MSCs as well as their anti-inflammatory properties [89]. In this study, the NASH-induced mice showed high levels of $\alpha$ SMA, a marker of hepatic stellate cell activation, collagen accumulation and high levels of inflammatory markers, like $\mathrm{TNF} \alpha$. But in NASH-induced mice treated with human MSc transplantation, collagen accumulation was decreased as well as $\alpha$-SMA expression, suggesting reversion of activated stellate cells to a quiescent state and a decrease in their fibrogenic potential. TNF $\alpha$ levels were also reduced, indicating a decrease in inflammation.

NASH is characterized by fat deposition in the liver, fibrosis, and inflammation, and is linked to metabolic syndrome and obesity. Due to the recent development of effective therapies for hepatitis $\mathrm{C}$ viral (HCV) infection as well as the steady increase of obesity in the Western world, it is expected that NASH will replace $\mathrm{HCV}$ as the most common chronic liver disease and indication for OLT within the next several years. The development of animal models of NASH, which are diet induced, is an important and crucial contribution to 
understanding the biology of the disease as well as the development of potential therapies [90]. Multiple studies have demonstrated the therapeutic benefit of HT in mouse and rat models of NASH [91-93]. In each of these studies, HT of either adult hepatocytes or MSCs has led to higher survival rates of animals, lower lipid stores in the liver, and improved liver function.

But perhaps the most promising and exciting development of cell therapy for CLD has been with iPS cells. In work by Liu et al., iPS cells were derived from cells from each of the three developmental germ layers (hepatocytes, fibroblasts, keratinocytes) and differentiated into hepatocyte-like cells. These cells secreted liver-specific proteins with morphology similar to adult hepatocytes. The differentiated hepatocyte-like cells were transplanted into an immunodeficient mouse model with DMNinduced liver fibrosis and were capable of engrafting into the liver (8-15\%) and regenerating the damaged liver regardless of germ layer origin. This is exciting for all the aforementioned benefits of using iPS cells as an alternative cell source for HT, but more specifically because it appears that the epigenetic differences between iPS cells derived from different developmental layers does not affect their ability to differentiate into similarly functional hepatocyte-like cells $[94 \bullet \bullet]$.

\section{Conclusion}

Hepatocyte transplantation research and clinical application has made great strides over recent years. The investigation into various cell sources and the use of animal models of liver disease have provided tools to justify the use of cell-based therapies when whole organ transplantation is not an option. There are still many hurdles to overcome, but increased knowledge and understanding of the liver's intrinsic ability to regenerate and discoveries harnessing the regenerative potential of hepatocytes and hepatocyte-like cells has the potential to significantly transform the practice of health care and the course of treatment of liver diseases in the near future.

\footnotetext{
Acknowledgments We thank the support of the Zell Family Foundation. J.R.F. acknowledges support from the National Institute of General Medical Sciences of the National Institutes of Health under Award Number T32GM105538. J.A.W. acknowledges support from the Robert R. McCormick Foundation, American Association for the Study of Liver Diseases and the American Liver Foundation's Liver Scholar Award, Society for Surgery for the Alimentary Tract's Career Development Award, and NIDDK K08DK10175. This work was supported in part by Health Resources and Services Administration contract 234-2005$37011 \mathrm{C}$. The content is the responsibility of the authors alone and does not necessarily reflect the views or policies of the Department of Health and Human Services, nor does mention of trade names, commercial products, or organizations imply endorsement by the U.S. Government.
}

\section{Compliance with Ethics Guidelines}

Conflict of Interest Jennifer R. Ferrer and Attasit Chokechanachaisakul declare that they have no conflict of interest.

Jason A. Wertheim reports non-financial support from Cellular Dynamics International, unrelated to the preparation of this manuscript.

Human and Animal Rights and Informed Consent This article does not contain any studies with human or animal subjects performed by any of the authors.

\section{References}

Papers of particular interest, published recently, have been highlighted as:

- Of importance

•• Of major importance

1. Ito M, Nagata G, Miyakawa S, Fox IJ. Review of hepatocyte transplantation. J Hepatobiliary Pancreatic Surg. 2009;6(2):97-100.

2. Fox IJ, Roy-Chowdhury J. Hepatocyte transplantation. J Hepatol. 2004;40(6):878-86.

3. Nussler AK, Zeilinger K, Schyschka L, et al. Cell therapeutic options in liver diseases: cell types, medical devices and regulatory issues. J Mater Sci Mater Med. 2011;22:1087-99.

4. Fox IJ, Chowdhury JR, Kaufman SS, et al. Treatment of CriglerNajjar syndrome type I with hepatocyte transplantation. N Engl J Med. 1998;338(20):1422-6.

5. Baccarani U, Adani GL, Sanna A, et al. Portal vein thrombosis after intraportal hepatocytes transplantation in a liver transplant recipient. Transpl Intl. 2005;18(6):750-4.

6. Fisher RA, Strom SC. Human hepatocyte transplantation: worldwide results. Transplantation. 2006;82(4):441-9.

7. Hughes RD, Mitry RR, Dhawan A. Current status of hepatocyte transplantation. Transplantation. 2012;93:342-7.

8. Hansel MC, Gramignoli R, Skvorak KJ, et al. The history and use of human hepatocytes for the treatment of liver diseases: the first 100 patients. Curr Protoc Toxicol. 2014;62:14.12.1-14.12.23.

9. Kim WR, Smith JM, SKeans MA, et al. OPTN/SRTR 2012 Annual data report: liver. Am J Transplant. 2014;1:69-96.

10. Dhawan A, Puppi J, Hughes RD, et al. Human hepatocyte transplantation: current experience and future challenges. Nature. 2012;7:288-98.

11. Dhawan A, Mitry RR, Hughes RD. Hepatocyte transplantation for liver-based metabolic disorders. J Inherit Metab Dis. 2006;29: 431-5.

12. Lee KW, Lee JH, Shin SW, et al. Hepatocyte transplantation for glycogen storage disease type 1b. Cell Transplant. 2007;16:629-37.

13. Mazariegos G, Shneider B, Burton B, et al. Liver transplantation for pediatric metabolic disease. Mol Genet Metab. 2014;111:418-27.

14. Shi M, Zhang Z, Xu R, et al. Human mesenchymal stem cell transfusion is safe and improves liver function in acute-on-chronic liver failure patients. Stem Cells Transl Med. 2012;1:725-31.

15. Gramignoli R, Dorko K, Tahan V, et al. Hypothermic storage of human hepatocytes for transplantation. Cell Transplant. 2014;23: 1143-51.

16. Pless G, Sauer IM, Rauen U. Improvement of the cold storage of isolated human hepatocytes. Cell Transplant. 2012;21(1):23-37.

17. Roll GF, Willenbring H. Transplanted nonviable human hepatocytes produce appreciable serum albumin levels in mice. Stem Cell Res. 2010;5:267-70. 
18. Puppi J, Strom SC, Hughes RD, et al. Improving the techniques for human hepatocyte transplantation: report from a consensus meeting in London. Cell Transplant. 2012;21:1-10.

19. Li T, Zhu J, Ma K, et al. Autologous bone marrow-derived mesenchymal stem cell transplantation promotes liver regeneration after portal vein embolization in cirrhotic rats. J Surg Res. 2013;182(2): 1161-73.

20. Yamanouchi K, Zhou H, Roy-Chowdhury N, et al. Hepatic irradiation augments engraftment of donor cells following hepatocyte transplantation. Hepatology. 2009;49:258-67.

21. Serra MP, Marongiu F, Sini M, et al. Hepatocyte senescence induced by radiation and partial hepatectomy in rat liver. Int $\mathrm{J}$ Radiat Biol. 2014;90(10):876-83.

22. Navarro-Alvarez N, Soto-Gutierrez A, Chen Y, et al. Intramuscular transplantation of engineered hepatic tissue constructs corrects acute and chronic liver failure in mice. J Hep. 2010;52:211-9.

23. Wang G, Zhou L, Ma X, et al. Monitoring of intrasplenic hepatocyte transplantation for acute-on-chronic liver failure: a prospective fiveyear follow-up study. Transpl Proc. 2014;46:192-8. This article describes a long-term study following 7 patients with ACLF. Patients received an intrasplenic hepatocyte transplantation and were followed for 5 years or until death. Three of the seven patients completely recovered from liver failure, one survived the time frame and underwent OLT, and three patients died within 12 months. There have not been any/many studies that have followed patients for this extent of time.

24. Zhou H, Dong X, Kabarriti R, et al. Single liver lobe repopulation with wildtype hepatocytes using regional hepatic irradiation cures jaundice in Gunn rats. Plos One. 2012;7(10):1-7.

25. Kamimura R, Ishii T, Sasaki N, et al. Comparative study of transplantation of hepatocytes at various differentiation stages into mice with lethal liver damage. Cell Transplant. 2012;21:2351-62.

26. Garmignoli R, Tahan V, Dorko K, et al. New potential cell source for hepatocyte transplantation: discarded livers from metabolic disease liver transplants. Stem Cell Res. 2013;11:563-73.

27. Meng FY, Liu L, Yang FH, et al. Reversible immortalization of human hepatocytes mediated by retroviral transfer and sitespecific recombination. World J Gastroenterol. 2014;20(36): 13119-26.

28. Fausto N, Campbell JS. The role of hepatocytes and oval cells in liver regeneration and repopulation. Mech Dev. 2003;120:117-30.

29. Chen Y, Zhou H, Sarver AL, et al. Hepatic differentiation of liverderived progenitor cells and their characterization by microRNA analysis. Liver Transpl. 2010;16:1086-97.

30. Shupe TD, Piscaglia AC, $\mathrm{Oh} \mathrm{SH}$, et al. Isolation and characterization of hepatic stem cells, or "oval cells", from rat livers. Methods Mol Biol. 2009;482:387-405.

31. Dollé L, Best J, Empsen C, et al. Successful isolation of liver progenitor cells by aldehyde dehydrogenase activity in naïve mice. Hepatology. 2012;55(2):540-52.

32. Tarlow BD, Pelz C, Naugler WE, et al. Bipotential adult liver progenitors are derived from chronically injured mature hepatocytes. Cell Stem Cell. 2014;15:605-18. This paper is significant because it elucidates the origin of the so-called hepatic stem cell population.

33. Schaub JR, Malato Y, Gormond C, et al. Evidence against a stem cell origin of new hepatocytes in a common mouse model of chronic liver injury. Cell Rep. 2014;8(4):933-9.

34. Yanger K, Kngin D, Zong Y, et al. Adult hepatocytes are generated by self-duplication rather than stem cell differentiation. Cell Stem Cell. 2014;15(3):340-9.

35. Pittenger MG, Mackay AM, Beck SC, et al. Multilineage potential of adult human mesenchymal stem cells. Science. 1999;284:143-7.

36. Kakinuma S, Tanaka Y, Chinzei R, et al. Human umbilical cord blood as a source of transplantable hepatic progenitor cells. Stem Cells. 2003;21:217-27.
37. De Coppi P, Bartsch Jr G, Siddiqui MM, et al. Isolation of amniotic stem cell lines with potential for therapy. Nat Biotechnol. 2007;25: $100-6$.

38. Yin L, Zhu Y, Yang J, et al.: Adipose tissue-derived mesenchymal stem cells differentiated into hepatocyte-like cell in vivo and in vitro. Molec Med Rep. 2014. 1-11.

39. Maijenburg MW, van der Schoot CE, Voermans C. Mesenchymal stromal cell migration: possibilities to improve cellular therapy. Stem Cell Dev. 2012;21:19-29.

40. Zuk PA, Zhu M, Mizuno H, et al. Multilineage cells from human adipose tissue: implications for cell-based therapies. Tissue Eng. 2001;7:211-28.

41. Yu J, Cao H, Yang J, et al. In vivo hepatic differentiation of mesenchymal stem cells from human umbilical cord blood after transplantation into mice with liver injury. Biochem Biophys Res Comm. 2012;422:539-45.

42. Amer MEM, El-Sayed SZ, El-Kheir WA, et al. Clinical and laboratory evaluation of patients with end-stage liver cell failure injected with bone marrow-derived hepatocyte-like cells. Euro J Gastroenterol Hepatol. 2011;23:936-41.

43. Takashi K, Tanabe K, Ohnuki M, et al. Induction of pluripotent stem cells from adult human fibroblasts by defined factors. Cell. 2007;131:861-72.

44. Kajiwara M, Aoi T, Okita K, et al. Donor-dependence variations in hepatic differentiation from human-induced pluripotent stem cells. Proc Natl Acad Sci U S A. 2012;109:12538-43.

45. Si-Tayeb K, Noto FK, Nagaoka M, et al. Highly efficient generation of human hepatocyte-like cells from induced pluripotent stem cells. Hepatology. 2010;51:297-305.

46. Grompe M, al-Dhalimy M, Finegold M, et al. Loss of fumarylacetoacetate hydrolase is responsible for the neonatal hepatic dysfunction phenotype of lethal albino mice. Genes Dev. 1993;7(12A):2298-307.

47. Espejel S, Roll GR, McLaughlin J, et al. Induced pluripotent-stem cell-derived hepatocytes have the functional and proliferative capabilities needed for liver regeneration in mice. J Clin Investig. 2010;120(9):3120-6.

48. Zhu S, Rezvani M, Harbell J, et al. Mouse liver repopulation with hepatocytes generated from human fibroblasts. Nature. 2014;508: 93-7. This study demonstrates the potential of human induced pluripotent cells to regenerate liver tissue.

49. Takayama K, Inamura M, Kawabata K, et al. Efficient generation of functional hepatocytes from human embryonic stem cells and induced pluripotent stem cells by HNF4a transduction. Mol Ther. 2012;20:127-37.

50. DeLaForest A, Nagaoka M, Si-Tayeb K, et al. HNF4A is essential for specification of hepatic progenitors from human pluripotent stem cells. Development. 2011;138:4143-53.

51. Tomizawa M, Shinozaki F, Sugiyama T, et al. Single-step protocol for the differentiation of human-induced pluripotent stem cells into hepatic progenitor-like cells. Biomed Reports. 2013;1:18-22.

52. Yang $\mathrm{T}, \mathrm{Li} \mathrm{C}$, Zhang L, et al. A promising hepatocyte-like cell line, CCL-13, exhibits good liver function both in vitro and in an acute liver failure model. Transpl Proc. 2013;45:688-94.

53. Basma H, Soto-Gutierrez A, Yannam GR, et al. Differentation and transplantation of human embryonic stem cell-derived hepatocytes. Gastroenterology. 2009;136(3):990-9.

54. Espejel S, Eckardt S, Harbell J, et al. Brief report: parthenogenetic embryonic stem cells are an effective cell source for therapeutic liver repopulation. Stem Cells. 2014;32:1983-8.

55. Nagata $\mathrm{H}$, Ito $\mathrm{M}$, Cai J, et al. Treatment of cirrhosis and liver failure in rats by hepatocyte xenotransplantation. Gastroenterology. 2003; 124(2):422-31.

56. Nagata H, Nashitai R, Shirota C, et al. Prolonged survival of porcine hepatocytes in cynomolgus monkeys. Gastroenterology. 2007;132(1):321-9. 
57. Schultz LD, Ishikawa F, Greiner DL. Humanized mice in translational biomedical research. Nat Rev Immunol. 2007;7:118-30.

58. Wilson EM, Bial J, Tarlow B, et al.: Extensive double humanization of both liver and hematopoiesis in FRGN mice. Stem Cell Res. 2014; 404-12

59. Gunn $\mathrm{CH}$. Hereditary acholuric jaundice in a new mutant strain of rats. J Heredity. 1938;29:137-9.

60. Gunn CH. Hereditary acholuric jaundice in the rat. Can M A J. 1944;50:230-7.

61. Watanabe Y. Serial inbreeding of rabbits with hereditary hyperlipidemia (WHHL-rabbit. Atherosclerosis. 1980;36(2):261-8.

62. Grompe M, Al-Halimy M, Finegold M, et al. Loss of fumarylacetoacetate hydrolase is responsible for the neonatal hepatic dysfunction phenotype of lethal albino mice. Genes Dev. 1993;7:2297-307.

63.• Hickey RD, Mao SA, Glorioso J, et al. Fumarylacetoacetate hydrolase deficient pigs are a novel large animal model of metabolic liver disease. Stem Cell Res. 2014;13:144-53. This paper describes the development of a large animal model of metabolic and acute liver disease.

64. Maerckx C, Tondreau T, Berardis S, et al. Human liver stem/ progenitor cells decrease serum bilirubin in hyperbilirubinemic Gunn rat. World J Gastroenterol. 2014;20(30):10553-63.

65. Hay DC. Cadaveric hepatocytes repopulate diseased livers: life after death. Gastroenterol. 2010;139(3):729-31.

66. Eggenhofer E, Popp FC, Renner P, et al. Allogeneic bone marrow transplantation restores liver function in Fah-knockout mice. Exp Hematol. 2008;36(11):1507-13.

67. Azuma H, Paulk N, Ranade A, et al. Robust expansion of human hepatocytes in Fah-/-/Rag2-/-/Il2rg-/- mice. Nat Biotechnol. 2007;25(8):903-10.

68. Yu B, He ZY, You P, et al. Reprogramming fibroblasts into bipotential hepatic stem cells by defined factors. Cell Stem Cell. 2013;13:328-40.

69. Wu G, Liu N, Rittelmeyer I, et al. Generation of healthy mice from gene-corrected disease-specific induced pluripotent stem cells. PLoS Biol. 2011;9(7):1-14.

70. Ding J, Yannam GR, Roy-Chowdhury N, et al. Spontaneous hepatic repopulation in transgenic mice expressing mutant human $\alpha 1$ antitrypsin by wild-type donor hepatocytes. J Clin Invest. 2011;121(5):1930-4.

71. Yusa K, Rashid ST, Strick-Marchand H, et al. Targeted gene correction of $\alpha 1$-antitrypsin deficiency in induced pluripotent stem cells. Nature. 2011;478(7369):391-4.

72. Allen KJ, Cheah DM, Wright PF, et al. Liver cell transplantation leads to repopulation and functional correction in a mouse model of Wilson's disease. J Gastroenterol Hepatol. 2004;19(11):1283-90.

73. Allen KJ, Cheah DM, Lee XL, et al. The potential of bone marrow stem cells to correct liver dysfunction in a mouse model of Wilson's disease. Cell Transplant. 2004;13(7-8):765-73.

74. Hamman KJ, Winn SR, Harding CO. Hepatocytes from wild-type or heterozygous donors are equally effective in achieving successful therapeutic liver repopulation in murine phenylketonuria (PKU). Mol Genet Metab. 2011;104(3):235-40.

75. Soltys KA, Soto-Gutierrez A, Nagaya M, et al. Barriers to the successful treatment of liver disease by hepatocyte transplantation. J Hepatol. 2010;53(4):769-74.

76. Sun K, Xie X, Xie J, et al. Cell-based therapy for acute and chronic liver failures: distinct diseases, different choices. Sci Rep. 2014;4: 6494.

77. Sun L, Fan X, Zhang L, et al. Bone mesenchmal stem cell transplantation via four routes for the treatment of acute liver failure in rats. Int J Molec Med. 2014;34:987-96.
78. Sauer V, Siaj R, Stöppeler S, et al. Repeated transplantation of hepatocytes prevents fulminant hepatitis in a rat model of Wilson's Disease. Liver Transpl. 2012;18(2):248-59.

79. Rahman TM, Hodgson HJF. Animal models of acute hepatic failure. Int J Exp Path. 2000;81:145-57.

80. Bélanger M, Butterworth RF. Acute liver failure: a critical appraisal of available animal models. Metab Brain Dis. 2005;20(4):409-23.

81. Li J, Zhang L, Xin J, et al. Immediate intraportal transplantation of human bone marrow mesenchymal stem cells prevents death from fulminant hepatic failure in pigs. Hepatology. 2012;56(3):1044-52.

This study describes a procedure for injecting bone marrow mesenchymal stem cells into a large animal model of acute liver disease that can potentially be translated into a future clinical therapy.

82. Strom SC, Chowdhury JR, Fox IJ. Hepatocyte transplantation for the treatment of human disease. Semin Liver Dis. 1999;19:39-48.

83. Weber A, Grover-Picard MT, Franco D, et al. Hepatocyte transplantation in animal models. Liver Transpl. 2009;15:7-14.

84. Hoppo T, Komori J, Manohar R, et al. Rescue of lethal hepatic failure by hepatized lymph nodes in mice. Gastroenterol. 2010;140(2):656-66.

85. Liedtke C, Luedde T, Sauerbruch T, et al. Experimental liver fibrosis research: update on animal models, legal issues and translational aspects. Fibrogenesis Tiss Repair. 2013;6:19-42.

86. Liu Y, Meyer C, Xu C, et al. Animal models of chronic liver diseases. Am J Physiol Gastointest Liver Physiol. 2013;304:G449-68.

87. Li T, Zhu J, Ma K, et al. Autologous bone marrow-derived mesenchymal stem cell transplantation promotes liver regeneration after portal vein embolization in cirrhotic rats. J Surg Res. 2013;84: 1161-73.

88. Piryaei A, Valojerdi MR, Shahsavani M, et al. Differentiation of bone marrow-derived mesenchymal stem cells into hepatocyte0like cells on nanofibers and their transplantation into a carbon tetrachloride-induced liver fibrosis model. Stem Cell Rev Rep. 2011;7:103-18.

89. Winkler S, Borkham-Kamphorst E, Stock P, et al. Human mesenchymal stem cells towards non-alcoholic steatohepatitis in an immunodeficient mouse model. Exp Cell Res. 2014;326:230-9.

90. Nakae D, Yoshiji H, Mizumoto Y, et al. High incidence of hepatocellular carcinomas induced by a choline deficient L-amino acid defined diet in rats. Cancer Res. 1992;52:5042-5.

91. Pelz S, Stock P, Brückner S, et al. A methionine-choline-deficient diet elicits NASH in the immunodeficient mouse featuring a model for hepatic cell transplantation. Exp Cell Res. 2012;218:276-87.

92. Nakamura Y, Mizuguchi T, Tanimizu N, et al.: Preoperative hepatocyte transplantation improves the survival of rats with nonalcoholic steaotohepatitis-related cirrhosis after partial hepatectomy. Cell Transpl. 2013. E-pub ahead of print.

93. Nakamura Y, Mizuguchi T, Naoki T, et al. Preoperative hepatocyte transplantation improves survival of rats with nonalcoholic steatohepatitis-related cirrhosis after partial hepatectomy. Cell Transpl. 2014;23:1243-54.

94.• Liu H, Kim Y, Sharkis S, et al. In vivo liver regeneration potential of human induced pluripotent stem cells from diverse origins. Sci Transl Med. 2011;3(((82):82ra39. There is a large appeal to using patient-specific iPS cells, and this study shows that iPS cells can be derived from cells of any developmental layer with equal efficacy, and that despite differences in epigenetic signatures, differentiated iPS cells from different germ layers behave the same way. 